\title{
Utilitarian walking and walking as exercise among community- dwelling older adults: what factors influence it?
}

\section{Abstract}

Objective: to investigate the association between utilitarian walking and walking as exercise, and socio-demographic, clinical and functional covariates related to these walking types. Methods: a cross-sectional exploratory study was conducted with 148 older adults (aged 60 and over). Walking frequency and duration was assessed using the IPEQ-W (Incidental and Planned Exercise Questionnaire - Version W). Socio-demographic, clinical conditions, level of disability and mobility were also assessed. Types of walking were compared among the variables using the Mann-Whitney test and non-parametric Spearman rho correlations were used to investigate the association between the types of walking and the variables. Results: the participants performed a mean of $1.1( \pm 2.1) \mathrm{h} /$ week of walking as exercise and $2.2( \pm 2.3) \mathrm{h} /$ week of utilitarian walking. Older adults who had diabetes $(p=0.015)$ did fewer h/week of walking as exercise. Participants who were older $(p=0.014)$, reported poor self-rated health $(p<0.001)$, poor disability levels $(p<0.001)$, hypertension $(p=0.048)$, strokes $(p<0.001)$, heart disease $(p=0.026)$, urinary incontinence $(p<0.001)$, dizziness $(p=0.008)$, or sleep disorders $(p=0.042)$ spent fewer hours performing utilitarian walking. Correlations between the covariates and types of walking varied from very weak to weak. Conclusion: chronic diseases and unfavorable health conditions decreased walking time. Utilitarian walking was the most frequent type of walking performed by the older adults. Health care professionals and public policy managers should use utilitarian walking as a way of increasing levels of physical activity and to promote healthy aging.
Waléria Paixão de Limal $\mathbb{D}$

Camila Astolphi Lima' ${ }^{\mathbb{D}}$ Renato Barbosa dos Santos' 10 Wuber Jefferson de Souza Soares' ${ }^{1}$ Monica Rodrigues Perracinil ${ }^{\mathbb{D}}$

Keywords: Exercise. Health of the Elderly. Walking. 


\section{INTRODUCTION}

Physical activity is one of the key determinants for healthy ageing and plays an important role in reducing disability, mortality and preventing chronic diseases ${ }^{1}$. A total of 150 minutes per week of moderate to-vigorous physical activity is recommended, in bouts of 10 minutes or more ${ }^{2}$. Meeting and maintaining at least the minimum physical activity recommendations could prevent $45 \%$ of deaths associated with physical inactivity ${ }^{3}$. Studies show that older people are more inactive and do not follow such recommendations ${ }^{4,5}$, which are frequently perceived as unattainable, particularly for older adults who are disabled and frail ${ }^{6}$.

Older people spend around two thirds of their time in sedentary activities, one third in light activities and only $2.1 \%$ of their days in activities considered moderate to vigorous ${ }^{7}$. Furthermore, it has been observed that the older the age, the greater is the tendency to sedentary behavior and/or to performing light physical activities?

One tool for increasing the level of physical activity and minimizing sedentary behavior is encouragement from health care professionals for older persons to be more active in general. The orientation for older adults to add small amounts of physical activity on a daily basis ${ }^{6}$ and increase the time spent on low-intensity activities, such as light walking ${ }^{8}$ are among the recommended strategies. Walking is the most common physical activity performed by adults', with an energy expenditure ranging from 2.0 METs (Metabolic Equivalent of Task) per hour for slow walking indoors to $2.5 \mathrm{MET}$ for utility walking and 3.5 METs for walking as exercise ${ }^{10}$.

Walking is recognized as an entry-level physical activity as it is accessible, inexpensive and safer than other strenuous forms of exercise, and can be monitored and quantified ${ }^{11,12}$. Overall, nearly 30\% of adult men and $40 \%$ of women reported walking as a leisure-time physical activity ${ }^{9}$ and, one in three older people reported walking outside the home on at least five days per week ${ }^{13}$. Among older people, unhurried walking is the main physical activity in the previous 90 days $^{14}$.
Not only is walking considered a good light to moderate exercise, it also can be used as a means of simply getting from one place to another for utilitarian purposes, such as shopping and connecting to public transport. The distance to amenities, the type of neighborhood, socioeconomic characteristics and walkability influence utilitarian walking ${ }^{15}$.

The physical and mental health benefits of walking as a moderate intensity exercise are widely recognized. Light intensity physical activities, such as utilitarian walking, have shown positive effects on body composition parameters (lower body mass index and waist circumference), the reduction of C-reactive protein levels and insulin resistance, in addition to reducing the risk of mortality ${ }^{8,16}$.

There is a growing consensus regarding the importance of encouraging sedentary older people to perform activities of any intensity ${ }^{16,17}$. However, the factors related to types of walking among older adults have been little explored. Understanding the sociodemographic factors, health conditions and functionality that can influence types of walking can help health professionals prescribe and guide walking as a way to increase the level of physical activity of older adults. Therefore, the aim of the present study was to investigate the association between utilitarian walking and walking as exercise, and between sociodemographic, clinical and functional covariates and these walking types.

\section{METHODS}

A cross-sectional exploratory study with community-dwelling older adults, aged 60 years and over, was conducted. The participants were registered at two reference centers for older adults in the city of São Paulo, in the state of São Paulo, Brazil. The sample was selected by convenience and the older adults were invited to participate while in the waiting room for ambulatory consultations. Data were collected between 2013 and 2014 and the two evaluators were previously trained.

The present study received approval from the Human Research Ethics Committee of the Universidade Cidade de São Paulo (protocol number 
129.812). All the participants received explanations regarding the objectives and procedures of the study and agreed to participate by signing a statement of informed consent.

Participants were excluded if they had cognitive impairment (based on Mini Mental State Examination score, adjusted by schooling $)^{18}$, were unable to walk (temporary or permanent) or used a wheelchair, and had aphasia or any severe sensory dysfunction that impaired communication.

To assess physical activity level the self-reported Incidental and Planned Exercise Questionnaire - IPEQ-W for older adults ${ }^{19}$ was used. This questionnaire estimates the frequency and duration of several levels of planned (exercise class, home exercises, walking, etc.) and incidental (household tasks, utilitarian walking, etc.) physical activities. There is a version of the IPEQ, the WA, which assesses the same issues with a recall time of three months (IPEQ-WA).

The final score is the total duration in hours per week, which is obtained by multiplying frequency and duration. The IPEQ-W can be divided into the following subdomains: incidental activities, walking activities, planned activities, planned walking activities and sports activities. For the present study, the total time spent on planned walking and on incidental or utilitarian walking was calculated.

The test-retest reliability of the original validation study of the IPEQ-W was assessed by the intraclass correlation coefficient (ICC) between the scores obtained in the initial survey and after one-week of follow-up. The following ICCs were obtained: 0.77 for comparison of total score; 0.89 and 0.26 , respectively, for questions related to frequency and duration of walking as exercise; and 0.81 and 0.75 , for utilitarian walking ${ }^{19}$. The IPEQ-W validity for walking was ascertained using its correlation with average moderate- to vigorous-intensity physical activity (MVPA) at low and medium cut-off points from an accelerometer and was 0.26 and 0.35 , respectively ${ }^{20}$.

To assess the reliability of the Brazilian version of IPEQ-W, we conducted a study with a sub-sample of 50 older adults and used the ICC ${ }^{2,1}(95 \% \mathrm{CI})$ and the ICC ${ }^{3,1}(95 \% \mathrm{CI})$ to test the inter-rater and the test-retest reliability, respectively. The ICC (inter-rater reliability) comparing planned walking (frequency and duration) was 0.88 (0.79-0.93) and 0.89 (0.800.93). The ICC comparing utilitarian walking was 0.87 (0.78-0.93) and 0.75 (0.57-0.86). The ICC (testretest reliability) for planned walking (frequency and duration) was 0.86 (0.74-0.92) and 0.92 (0.86-0.95). The ICC (test-retest reliability) comparing utilitarian walking was 0.94 (0.89-0.96) and 0.82 (0.68-0.89). Overall, the test- retest and inter-rater reliability of the IPEQ-W ranged from substantial to excellent ${ }^{21}$.

To characterize the sample we used a semi structured questionnaire that included sociodemographic questions (age, sex, income and living alone), number of self-reported diseases, health conditions and the regular use of medications. The use of five or more medications on a regular basis was considered polypharmacy.

The presence of depressive symptoms was assessed using the Geriatric Depression Scale (GDS). This scale consists of 15 questions that track the mood of individuals over the previous week. A final score above 5 points was considered positive for depressive symptoms. The scale has good sensitivity $(81 \%)$ and specificity $(71 \%)^{22}$.

The level of disability was assessed using the World Health Organization disability assessment schedule (WHODAS 2.0) ${ }^{23}$. It is composed of 12 questions divided into six domains: cognition, mobility, self-care, getting along, life activities and participation. Each item ranging from 0 (no problem) to 4 (severe problem or unable to perform) was totaled. The total score ranges from 0 to 48 points, with a higher score indicating greater disability.

Mobility was assessed with the Timed Up-and-Go Test (TUGT) ${ }^{24}$, which measures the time it takes an individual to rise from a standard chair with armrests, walk a distance of $3 \mathrm{~m}$, turn, walk back to the chair and sit down. The participant was instructed to complete the task as quickly as possible, without running. The time spent to complete the task was computed, with a low time indicating better performance in the test. The reliability of the TUGT is 0.91 . 
To calculate the sample size, it was considered that rejecting the null hypothesis $(\mathrm{H} 0)$ with a value of $\beta=0.20$ and $\alpha=0.05$ and with an estimated correlation coefficient of $r=0.250$ would require a sample with 123 participants.

Descriptive analyses were carried out to characterize the sample. The average weekly hours of walking as exercise and utilitarian walking were compared in relation to the variables of interest using the Mann-Whitney test for variables with two categories and the Kruskal Wallis test for variables with three or more categories. Post hoc analysis was performed using pairwise comparisons, with the adjusted Kruskal Wallis test. Spearman rho's nonparametric correlations were calculated to investigate the association between walking as exercise and utilitarian walking and the variables of interest.

The criteria suggested by Schober et al. (2018) ${ }^{25}$ for evaluating correlation strength is $00-0.10$ (negligible),
0.10-0.30 (weak), 0.40-0.69 (moderate), 0.70-0.89 (strong) or 0.90-1.0 (very strong).

\section{RESULTS}

One hundred and forty eight older adults were included in this study (Figure 1). The average scores of participants were $10.4( \pm 3.5)$ seconds for the TUGT, $2.9( \pm 2.9)$ points for the GDS and 2.04 .7 $( \pm 5.7)$ points for the WHODAS. The participants performed an average of $1.0( \pm 2.1)$ hours per week of walking as exercise, ranging from 0 to 10.5 hours and, on average $2.2( \pm 2.3)$ hours per week of utilitarian walking, ranging from 0 to 13.5 hours.

The comparison between covariates and hours per week of walking as exercise and utilitarian walking is shown in table 1 . The correlation between walking as exercise and utilitarian walking and the variables of interest is shown in tables 2 and 3 .

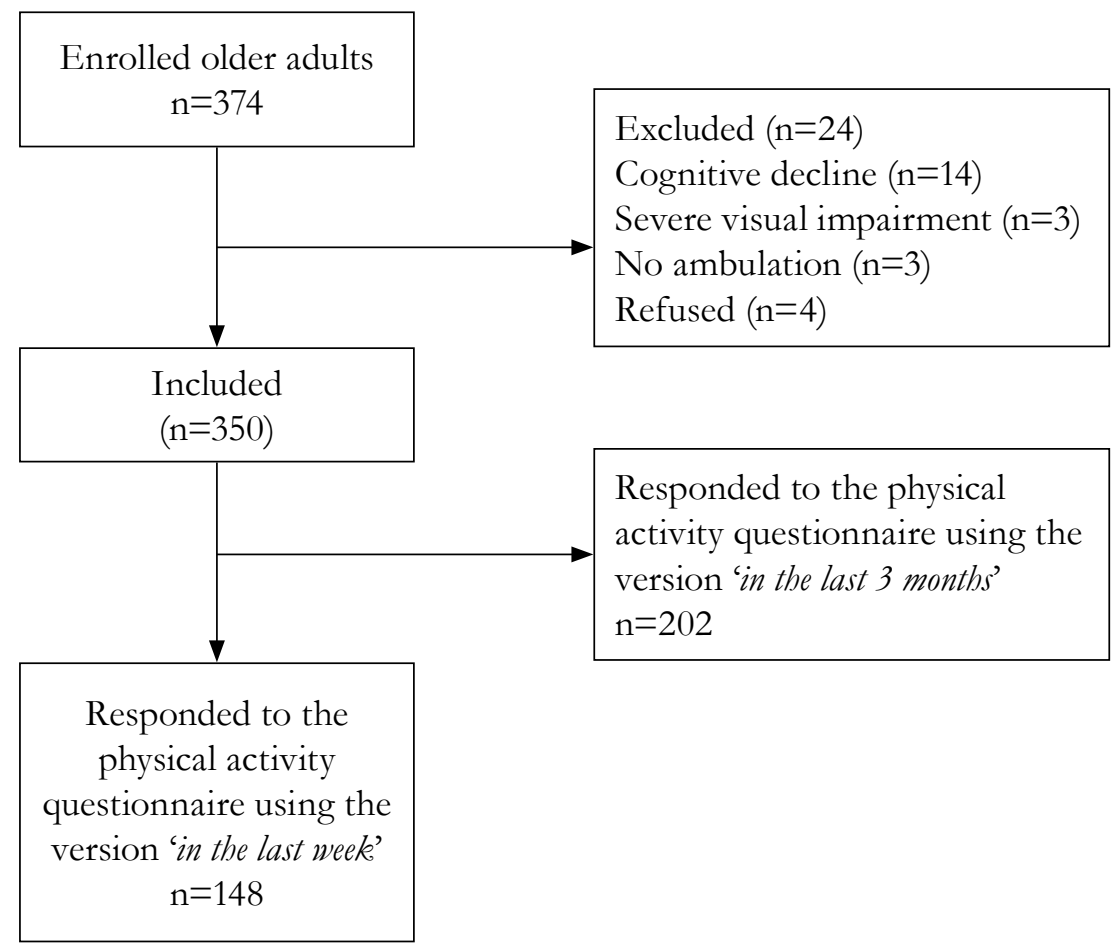

Figure 1. Study flowchart of participants who responded to the IPEQ-W (in the last week). Older adults who responded to the IPEQ-WA (in the last 3 months) were excluded. São Paulo, 2014. 
Table 1. Comparison between walking as exercise and utilitarian walking in older adults receiving follow-up care at a reference center for older people, São Paulo, 2014 (N=148).

\begin{tabular}{|c|c|c|c|c|c|}
\hline \multirow[t]{2}{*}{ Variables } & \multirow{2}{*}{$\begin{array}{l}\text { Total } \\
\text { population } \\
\mathrm{N}(\%) \\
\end{array}$} & \multicolumn{3}{|c|}{$\begin{array}{c}\text { Walking (hours/week) } \\
\text { Mean }(95 \% \mathrm{CI})\end{array}$} & \multirow[b]{2}{*}{$p$-value } \\
\hline & & Exercise & $p$-value & Utilitarian & \\
\hline \multicolumn{6}{|l|}{ Age } \\
\hline $60-74$ & $103(69.6)$ & $1.2(0.7-1.6)$ & 0.132 & $2.4(1.9-3.0)$ & 0.014 \\
\hline $75+$ & $45(30.4)$ & $0.7(0.2-1.1)$ & & $1.6(1.2-2.0)$ & \\
\hline \multicolumn{6}{|l|}{ Sex } \\
\hline Women & $126(85.1)$ & $1.1(0.7-1.5)$ & 0.343 & $2.2(1.8-2.7)$ & 0.563 \\
\hline Men & $22(14.9)$ & $0.6(0.0-1.3)$ & & $1.9(1.1-2.7)$ & \\
\hline \multicolumn{6}{|l|}{ Income (in R\$) } \\
\hline $0.0-1.0$ & $11(7.4)$ & $1.3(0.0-3.4)$ & 0.926 & $1.5(0.9-2.2)$ & 0.757 \\
\hline $1.1-2.0$ & $98(66.2)$ & $0.9(0.5-1.3)$ & & $2.2(1.6-2.7)$ & \\
\hline $2.1-3.0$ & $20(13.5)$ & $1.1(0.1-2.0)$ & & $2.3(1.4-3.2)$ & \\
\hline $3.1+$ & $19(12.8)$ & $1.2(0.0-2.5)$ & & $2.5(1.6-3.3)$ & \\
\hline \multicolumn{6}{|l|}{ Living alone } \\
\hline Yes & $38(25.7)$ & $1.3(0.6-1.9)$ & 0.397 & $2.6(1.6-3.6)$ & 0.199 \\
\hline No & $110(74.3)$ & $0.9(0.5-1.3)$ & & $2.0(1.7-2.4)$ & \\
\hline Stroke & & & 0.631 & & $<0.001$ \\
\hline Yes & $11(7.4)$ & $0.7(0.3-1.8)$ & & $0.9(0.4-1.4)$ & \\
\hline No & $137(92.6)$ & $1.0(0.7-1.4)$ & & $2.3(1.9-2.7)$ & \\
\hline Depression & & & 0.791 & & 0.403 \\
\hline Yes & $36(24.3)$ & $0.9(0.2-1.7)$ & & $1.7(0.9-2.5)$ & \\
\hline No & $112(75.7)$ & $1.0(0.6-1.4)$ & & $2.3(1.9-2.8)$ & \\
\hline Hypertension & & & 0.177 & & 0.048 \\
\hline Yes & $112(75.7)$ & $0.9(0.5-1.3)$ & & $2.0(1.6-2.4)$ & \\
\hline No & $36(24.3)$ & $1.4(0.6-2.3)$ & & $2.9(1.9-3.8)$ & \\
\hline Diabetes & & & 0.015 & & 0.602 \\
\hline Yes & $59(39.9)$ & $0.5(0.2-0.9)$ & & $2.1(1.4-2.7)$ & \\
\hline No & $89(60.1)$ & $1.3(0.8-1.8)$ & & $2.3(1.8-2.8)$ & \\
\hline Heart disease & & & 0.675 & & 0.026 \\
\hline Yes & $28(18.9)$ & $0.8(0.2-1.8)$ & & $1.5(0.9-2.1)$ & \\
\hline No & $120(81.1)$ & $1.0(0.7-1.4)$ & & $2.3(1.9-2.8)$ & \\
\hline Osteoarthritis & & & 0.926 & & 0.827 \\
\hline Yes & 28 (18.9) & $0.7(0.3-1.8)$ & & $0.9(0.4-1.4)$ & \\
\hline No & $120(81.1)$ & $1.0(0.6-1.4)$ & & $2.3(1.9-2.7)$ & \\
\hline Urinary incontinence & & & 0.481 & & $<0.001$ \\
\hline Yes & $28(18.9)$ & $0.7(0.3-1.8)$ & & $1.1(0.6-1.6)$ & \\
\hline No & $120(81.1)$ & $0.9(0.6-1.7)$ & & $2.4(2.0-2.9)$ & \\
\hline Dizziness & & & 0.076 & & 0.008 \\
\hline Yes & $46(31.1)$ & $0.6(0.2-1.1)$ & & $1.6(1.1-2.0)$ & \\
\hline No & $102(68.9)$ & $1.2(0.7-1.6)$ & & $2.5(1.9-3.0)$ & \\
\hline
\end{tabular}


Continuation of Table 1

\begin{tabular}{|c|c|c|c|c|c|}
\hline \multirow[t]{2}{*}{ Variables } & \multirow{2}{*}{$\begin{array}{l}\text { Total } \\
\text { population } \\
\mathrm{N}(\%)\end{array}$} & \multicolumn{3}{|c|}{$\begin{array}{c}\text { Walking (hours/week) } \\
\text { Mean }(95 \% \mathrm{CI})\end{array}$} & \multirow[b]{2}{*}{$p$-value } \\
\hline & & Exercise & $p$-value & Utilitarian & \\
\hline Sleep problems & & & 0.671 & & 0.042 \\
\hline Yes & $34(23.0)$ & $1.1(0.3-2.0)$ & & $1.6(1.1-2.2)$ & \\
\hline No & $114(77.0)$ & $1.0(0.6-1.4)$ & & $2.4(1.9-2.8)$ & \\
\hline Number of comorbidities & & & 0.101 & & 0.142 \\
\hline 0 & $15(10.1)$ & $2.0(0.2-3.8)$ & & $3.1(1.5-4.7)$ & \\
\hline $1-2$ & $69(46.7)$ & $1.2(0.6-1.7)$ & & $2.5(1.8-3.1)$ & \\
\hline 3 or more & $64(43.2)$ & $0.6(0.2-1.0)$ & & $1.6(1.2-2.0)$ & \\
\hline $\begin{array}{l}\text { Polypharmacy } \\
(\geq 5)\end{array}$ & & & 0.283 & & 0.019 \\
\hline Yes & $43(29.1)$ & $0.8(0.1-1.4)$ & & $1.5(1.0-2.0)$ & \\
\hline No & $105(70.9)$ & $1.1(0.7-1.5)$ & & $2.5(2.0-3.0)$ & \\
\hline Perceived health & & & 0.070 & & $<0.001$ \\
\hline Very good and good & $76(51.4)$ & $1.4(0.8-2.0)$ & & $2.8(2.2-3.4)^{\mathrm{a}}$ & \\
\hline Fair & $61(41.2)$ & $0.6(0.2-1.0)$ & & $1.6(1.2-2.0)^{\mathrm{b}}$ & \\
\hline Very poor and poor & $11(7.4)$ & $0.5(0.0-1.1)$ & & $1.2(0.0-2.5)^{\mathrm{c}}$ & \\
\hline WHODAS 2.0 (0-12) & & & 0.052 & & $<0.001$ \\
\hline $1^{\text {st }}$ tercile & $58(39.2)$ & $1.3(0.7-1.9)$ & & $3.3(2.5-4.1)^{d}$ & \\
\hline $2^{\text {nd }}$ tercile & $42(28.4)$ & $1.4(0.5-2.3)$ & & $1.7(1.2-2.1)^{\mathrm{e}}$ & \\
\hline $3^{\text {rd }}$ tercile & $48(32.4)$ & $0.3(0.0-0.5)$ & & $1.3(0.9-1.7)^{\mathrm{f}}$ & \\
\hline
\end{tabular}

Table 2. Correlation between walking as exercise and utilitarian walking and variables of interest in older adults receiving follow-up care at a reference center for older people, São Paulo, 2014 (N=148).

\begin{tabular}{|c|c|c|}
\hline \multirow{2}{*}{ Variables } & \multicolumn{2}{|c|}{ Walking } \\
\hline & Exercise & Utilitarian \\
\hline \multirow[t]{2}{*}{ Age, years } & $r h o=-0.131$ & $r h o=-0.171$ \\
\hline & $p=0.113$ & $p=0.037$ \\
\hline \multirow[t]{2}{*}{ Income, $\mathrm{R} \$$} & $r h o=0.075$ & $r h o=0.126$ \\
\hline & $p=0.363$ & $p=0.126$ \\
\hline \multirow[t]{2}{*}{ Education, years } & $r h o=0.111$ & $r h o=0.016$ \\
\hline & $p=0.179$ & $p=0.847$ \\
\hline \multirow[t]{2}{*}{ Number of medications } & $r h o=-0.105$ & $r h_{0}=-0.234$ \\
\hline & $p=0.203$ & $p=0.004$ \\
\hline \multirow[t]{2}{*}{ Number of comorbidities } & $r h o=-0.185$ & $r h o=-0.228$ \\
\hline & $p=0.025$ & $p=0.005$ \\
\hline \multirow[t]{2}{*}{ TUGT, seconds } & $r h o=-0.201$ & $r h o=-0.265$ \\
\hline & $p=0.014$ & $p=0.001$ \\
\hline \multirow{2}{*}{$\begin{array}{l}\text { WHODAS } 2.0 \text {, points } \\
(0-48)\end{array}$} & $r h o=-0.201$ & $r h o=-0.328$ \\
\hline & $p=0.014$ & $p<0.001$ \\
\hline \multirow{2}{*}{$\begin{array}{l}\text { GDS, points } \\
(0-15)\end{array}$} & $r h o=-0.174$ & $r h o=-0.201$ \\
\hline & $p=0.034$ & $p=0.014$ \\
\hline
\end{tabular}

Spearman rho correlation 
Table 3. Correlation between walking as exercise and utilitarian walking and WHODAS 2.0 domain scores in older adults receiving follow-up care at a reference center for older people, São Paulo, 2014 (N=148).

\begin{tabular}{|c|c|c|}
\hline \multirow{2}{*}{ Domains WHODAS 2.0} & \multicolumn{2}{|c|}{ Walking } \\
\hline & Exercise & Utilitarian \\
\hline \multirow[t]{2}{*}{ Cognitive } & $r h o=-0.192$ & $r h o=-0.207$ \\
\hline & $p=0.019$ & $p=0.008$ \\
\hline \multirow[t]{2}{*}{ Mobility } & $r h o=-0.159$ & $r b o=-0.273$ \\
\hline & $p=0.054$ & $p=0.001$ \\
\hline \multirow[t]{2}{*}{ Self-care } & rho $=-0.031$ & $r h o=-0.099$ \\
\hline & $p=0.711$ & $p=0.233$ \\
\hline \multirow[t]{2}{*}{ Relationship } & $r h o=-0.190$ & $r h o=-0.190$ \\
\hline & $p=0.021$ & $p=0.021$ \\
\hline \multirow[t]{2}{*}{ Life activities } & rho $=-0.174$ & $r h o=-0.214$ \\
\hline & $p=0.034$ & $p=0.009$ \\
\hline \multirow[t]{2}{*}{ Participation } & $r h o=-0.056$ & $r h o=-0.248$ \\
\hline & $p=0.498$ & $p=0.002$ \\
\hline
\end{tabular}

Spearman rho correlation

\section{DISCUSSION}

The current study investigated the association between sociodemographic, clinical and functional factors and different types of walking. The time in hours per week spent walking as an exercise was lower among older adults who reported having diabetes, and the time spent on utilitarian walking was lower among older adults who reported hypertension, heart disease, strokes, urinary incontinence, dizziness, sleep problems, polypharmacy, who self-rated their health as fair, bad or very bad and had a worse level of functionality. In general, the correlation between the covariates and the types of walking varied from very weak to weak.

The World Health Organization, in a study on Global Aging and Adult Health conducted in six low and middle-income countries (LMICs), revealed that low levels of physical activity $(<150$ minutes of moderate to vigorous intensity) were associated with physical health problems, worse self-reported health and comorbidities, such as chronic back pain, sleeping problems, visual and hearing problems, stroke and chronic obstructive pulmonary disease. In addition, there was a score of $3 \%$ per unit of disability score measured by WHODAS 2.0 for low physical activity $(\mathrm{OR}=1.03,95 \%$ CI 1.02-1.03).
In the present study, it was observed that of all diseases and health conditions, only diabetes negatively influenced walking as an exercise. Older people with diabetes spent less time walking as an exercise than older people without diabetes. This fact has also been observed in young people with diabetes (59.1 \pm 13.3 years), who were less active in terms of walking (with diabetes $90 \pm 225$ vs. without diabetes $135 \pm 275$ min week $)^{26}$. Factors that can prevent the participation of persons with diabetes in walking as exercise can include fear of hypoglycemia and problems related to peripheral neuropathies ${ }^{27}$. This result is highly unfavorable, since there is substantial evidence that structured aerobic exercise is effective in improving glycemic control in patients with type 2 diabetes $^{28}$.

Studies show the influence of different health conditions on walking. The results of the present study showed that the older people who reported having a disease and/or negative health condition engaged in less time of utilitarian walking. For example, older people who report urinary incontinence and dizziness generally have lower levels of habitual walking ${ }^{29}$, as they may feel uncomfortable and insecure when walking outdoors, due to the nature of the environmental and circumstantial unpredictability of activities outside the home. Furthermore, nearly 
$20 \%$ of older people reported dizziness while walking in the previous year, which may help to explain why they usually performed fewer hours per week of utilitarian walking ${ }^{30}$. Sleep problems, such as daytime sleepiness, also impair everyday activities, and are associated with poor self-rated health, obesity and depressive symptoms ${ }^{31}$.

Excessive medication use is associated with poor gait performance. Each additional medication taken increases the risk of gait decline by $12 \%$ to $16 \% \%^{32}$. The adverse effects of some medications, such as antihypertensive drugs, can cause dizziness and orthostatic hypotension. Some medications can affect balance and gait control, resulting in less willingness and confidence in relation to walking outside the home.

Disability level negatively influenced utilitarian walking. However, a negative and weak correlation between utilitarian walking and the mobility domain of the WHODAS 2.0. Studies have observed that the prevalence of older people who engaged in utilitarian walking decreased among those using walking aids ${ }^{33}$, poor balance, poor lower limb functionality and high disability levels in activities of daily living ${ }^{13}$. Having a functional limitation, particularly mobility limitation, can prevent people from walking.

The presence of comorbidities influenced the time spent on utilitarian walking more than the time spent on walking as exercise. However, the average number of hours per week of utilitarian walking was much higher than that spent on walking as exercise. This difference may have been due to the various barriers that older persons face when engaging in structured exercises, such as fear of suffering injuries, competing priorities, unsuitable climate, among others ${ }^{17}$. In contrast, utilitarian walking commonly occurs as part of activities of daily living, and is generally of low intensity, resulting in less discomfort. Almost $30 \%$ of older people walk for utilitarian purposes at least once a week ${ }^{13}$.

Environmental factors can directly influence the performance of different types of walking. Neighborhood characteristics, residential density, better mobility, access to destinations and services and availability of green spaces can be considered key factors. Short distances to access goods and services, such as a bus and metro stop, hospital, shops and supermarkets are important for encouraging older adults to engage in utilitarian walks ${ }^{13}$.

There is widespread recognition that health care practitioners play a critical role in increasing physical activity level amongst older adults, not only in increasing physical fitness but also in keeping older people as active as possible. Although exercise is a recognized treatment for multiple chronic conditions, its prescription is still neglected and commonly poorly addressed by clinicians. On its own, however, a recommendation to walk is insufficient if there is no personalized guidance and prescription, in addition to the care that should take into account barriers and facilitators over time ${ }^{17}$.

Older adults can become more active, combining walking as exercise and utilitarian walking ${ }^{13}$. Positive results were observed in groups who received guidance in walking ${ }^{34}$. In addition to setting goals, the development of skills to increase self-efficacy when carrying out utilitarian walking, such as carrying bags, avoiding obstacles and walking in places with a lot of people, seem to be promising interventions ${ }^{35}$.

Certain limitations of the present study should be acknowledged. Reported frequencies and duration of walking types are prone to recall bias and the investigation over a time frame of a week may not have captured a consistent pattern of walking activity. We interviewed older adults that were receiving follow-up care in two reference centers for older people that cover a specific geographical area of the city. However, these centers are located in large city, and are the referral points for approximately five million people with different socioeconomic and demographics profiles. The catchment area of the centres include various neighborhoods and built environments with different accessibility characteristics. While we interviewed a broad range of older people while they were waiting for medical, dental and rehabilitation appointments, they may not represent the entire population. Causal relationships could not be investigated due to the cross-sectional study design. Our sample size may not be sufficient to reject the null hypothesis for very weak correlations. 


\section{CONCLUSION}

Utilitarian walking is the most common walking type used by the older people in their activities of daily living and should be encouraged by health professionals and public health managers as a way of increasing levels of physical activity and promote healthy aging. Diseases and unfavorable health conditions negatively influence walking as a physical activity. Strategies for assessing, intervening and monitoring the health of older people should be implemented as a way of minimizing barriers to utilitarian walking. Future research to identify predictors of less time spent in utilitarian walking

\section{REFERENCES}

1. Bauman A, Merom D, Bull F, Buchner D, Fiatarone Singh M. Updating the evidence for physical activity: Summative Reviews of the Epidemiological Evidence, Prevalence, and Interventions to Promote "Active Aging”. Gerontologist. 2016;56 Suppl 2:268-80.

2. United States. Department of Health and Human Services. Physical activity for americans. 2nd. ed. Washington, DC: Department of Health and Human Service; 2018.

3. Mok A, Khaw KT, Luben R, Wareham N, Brage S. Physical activity trajectories and mortality: population based cohort study. BMJ. 2019;365:1-10.

4. Gobbi S, Sebastiao E, Papini CB, Nakamura PM, Valdanha Netto A, Gobbi LT, et al. Physical inactivity and related barriers: a study in a community dwelling of older brazilians. J Aging Res. 2012;2012:1-10.

5. Zaitune MP, Barros MB, Cesar CL, Carandina L, Goldbaum M, Alves MC. Factors associated with global and leisure-time physical activity in the elderly: a health survey in Sao Paulo (ISA-SP), Brazil. Cad Saúde Pública. 2010;26(8):1606-18.

6. Sparling P, Howard B, Dunstan D, Owen N. Recommendations for physical activity in older adults. BMJ. 2015;350:1-10.

7. Dos Santos CES, Manta SW, Maximiano GP, Confortin SC, Benedetti TRB, D’Orsi E, et al. Accelerometer-measured physical activity and sedentary behavior: a cross-sectional study of Brazilian Older Adults. J Phys Act Health. 2018;15(11):811-18. and intervention protocols to assess the effectiveness of utilitarian walking should be conducted.

\section{ACKNOWLEDGEMENTS}

The authors would like to thank the Centro de Referência do Idoso da Zona Norte (the North Zone Reference Center for Older Adults) (CRI) and Instituto Paulista de Geriatria e Gerontologia (the Sao Paulo Institute of Geriatrics and Gerontology) (IPGG) for collaborating in the data collection phase.

Edited by: Ana Carolina Lima Cavaletti
8. Loprinzi P, Lee H, Cardinal B. Evidence to support including lifestyle light-intensity recommendations in physical activity guidelines for older adults. Am J Health Promot. 2015;29(5):277-84.

9. Dai S, Carroll DD, Watson KB, Paul P, Carlson SA, Fulton JE. Participation in Types of Physical Activities Among US Adults--National Health and Nutrition Examination Survey 1999-2006. J Phys Act Health. 2015;12 Suppl 1:128-40.

10. Ainsworth BE, Haskell WL, Herrmann SD, Meckes N, Bassett Jr. DR, Tudor-Locke C, et al. Compendium of Physical Activities [Internet]. Arizona: Arizona States University; 2011 [acesso em 26 maio 2020]. Disponível em: https://sites.google.com/site/ compendiumofphysicalactivities/Activity-Categories

11. Hallal PC, Andersen LB, Bull FC, Guthold R, Haskell W, Ekelund U, et al. Global physical activity levels: surveillance progress, pitfalls, and prospects. Lancet. 2012;380(9838):247-57.

12. Sallis R, Matuszak J, Baggish A, Franklin B, Chodzko-Zajko W, Fletcher B, et al. Call to action on making physical activity assessment and prescription a medical standard of care. Curr Sports Med Rep. 2016;15(3):207-14.

13. Procter-Gray E, Leveille SG, Hannan MT, Cheng J, Kane K, Li W. Variations in Community Prevalence and Determinants of Recreational and Utilitarian Walking in Older Age. J Aging Res. 2015;2015:1-10. 
14. Ramalho JR, Lima-Costa MF, Firmo JO, Peixoto SV. Energy expenditure through physical activity in a population of community-dwelling Brazilian elderly: cross-sectional evidences from the Bambui Cohort Study of Aging. Cad Saúde Pública. 2011;27 Suppl 3:399-408.

15. Hajna S, Ross NA, Joseph L, Harper S and Dasgupta K. Neighbourhood walkability, daily steps and utilitarian walking in Canadian adults. BMJ Open. 2015;5(11):e008964 [10 p.].

16. Fuzeki E, Engeroff T, Banzer W. Health benefits of light-intensity physical activity: a systematic review of Accelerometer Data of the National Health and Nutrition Examination Survey (NHANES). Sports Med. 2017;47(9):1769-93.

17. Perracini MR, Franco MRC, Ricci NA, Blake C. Physical activity in older people - Case studies of how to make change happen. Best Pract Res Clin Rheumatol. 2017;31(2):260-74.

18. Bertolucci PH, Brucki SM, Campacci SR, Juliano Y. The Mini-Mental State Examination in a general population: impact of educational status. Arq Neuropsiquiatr. 1994;52(1):1-7.

19. Delbaere K, Hauer K, Lord SR. Evaluation of the incidental and planned activity questionnaire (IPEQ) for older people. Br J Sports Med. 2010;44(14):1029-34.

20. Merom D, Delbaere K, Cumming R, Voukelatos A, Rissel C, Van Der Ploeg HP, et al. Incidental and planned exercise questionnaire for seniors: validity and responsiveness. Med Sci Sports Exerc. 2014;46(5):947-54.

21. Landis JR, Koch GG. The measurement of observer agreement for categorical data. Biometrics. 1977;33:159-74.

22. Almeida OPA, Shirley A. Reliability of the brazilian version of the Geriatric Depression Scale (GDS) short form. Arq Neuropsiquiatr. 1999;57:421-6.

23. Ustun TB, Chatterji S, Kostanjsek N, Rehm J, Kennedy C, Epping-Jordan J, et al. Developing the World Health Organization Disability Assessment Schedule 2.0. Bull World Health Organ. 2010;88(11):815-23.

24. Podsiadlo D, Richardson S. The timed "Up \& Go": a test of basic functional mobility for frail elderly persons. J Am Geriatr Soc. 1991;39(2):142-8.

25. Schober P, Boer C, Schwarte LA. Correlation coefficients: appropriate use and interpretation. Anesth Analg. 2018;126(5):1763-8.
26. Huebschmann AG, Crane LA, Belansky ES, Scarbro S, Marshall JA, Regensteiner JG. Fear of injury with physical activity is greater in adults with diabetes than in adults without diabetes. Diabetes Care. 2011;34(8):1717-22.

27. Adeniyi AF, Anjana RM, Weber MB. Global account of barriers and facilitators of physical activity among patients with Diabetes Mellitus: a narrative review of the literature. Curr Diabetes Rev. 2016;12(4):440-8.

28. Delevatti RS, Bracht CG, Lisboa SDC, Costa RR, Marson EC, Netto N, et al. The Role of aerobic training variables progression on glycemic control of patients with Type 2 Diabetes: a systematic review with meta-analysis. Sports Med Open. 2019;5(1):1-10.

29. Lee AH, Hirayama F. Physical activity and urinary incontinence in older adults: a community-based study. Curr Aging Sci. 2012;5(1):35-40.

30. De Moraes SA, Soares WJ, Rodrigues RA, Fett WC, Ferriolli E, Perracini MR. Dizziness in communitydwelling older adults: a population-based study. Braz J Otorhinolaryngol. 2011;77(6):691-9.

31. Lima CA, Soares WJ, Bilton TL, Dias RC, Ferrioll E, Perracini MR. Correlates of excessive daytime sleepiness in community-dwelling older adults: an exploratory study. Rev Bras Epidemiol. 2015;18(3):607-17.

32. Montero-Odasso M, Sarquis-Adamson Y, Song HY, Bray NW, Pieruccini-Faria F, Speechley M. Polypharmacy, gait performance, and falls in community-dwelling oder adults: Results from the Gait and Brain Study. J Am Geriatr Soc. 2019;67(6):1182-8.

33. Winters M, Voss C, Ashe MC, Gutteridge K, McKay H, Sims-Gould J. Where do they go and how do they get there? Older adults' travel behaviour in a highly walkable environment. Soc Sci Med. 2015;133:304-12.

34. Kassavou A, Turner A, French DP. Do interventions to promote walking in groups increase physical activity?: a meta-analysis. Int J Behav Nutr Phys Act. 2013;10:1-8.

35. Salbach NM, Barclay R, Webber SC, Jones CA, Mayo NE, Lix LM, et al. A theory-based, taskoriented, outdoor walking programme for older adults with difficulty walking outdoors: protocol for the Getting Older Adults Outdoors (GOOUT) randomised controlled trial. BMJ Open. 2019;9(4):e029393 [10 p.]. 